\title{
How Much Is Your Strangle Worth? On the Relative Value of the $\delta$ - Strangle under the Black-Scholes Pricing Model
}

\author{
Ben Boukai ${ }^{1}$ \\ ${ }^{1}$ Department of Mathematical Sciences, IUPUI, Indianapolis, IN 46202, USA \\ Correspondence: Ben Boukai, Department of Mathematical Sciences, IUPUI, Indianapolis, IN 46202, USA.
}

Received: June 4, 2020

Accepted: June 22, $2020 \quad$ Available online: June 29, 2020

doi:10.11114/aef.v7i4.4887

URL: https://doi.org/10.11114/aef.v7i4.4887

\begin{abstract}
Trading option strangles is a highly popular strategy often used by market participants to mitigate volatility risks in their portfolios. We propose a measure of the relative value of a delta-Symmetric Strangle and compute it under the standard Black-Scholes-Merton option pricing model. This new measure accounts for the price of the strangle, relative to the Present Value of the spread between the two strikes, all expressed, after a natural re-parameterization, in terms of delta and a volatility parameter. We show that under the standard BSM model, this measure of relative value is bounded by a simple function of delta only and is independent of the time to expiry, the price of the underlying security or the prevailing volatility used in the pricing model. We demonstrate how this bound can be used as a quick benchmark to assess, regardless the market volatility, the duration of the contract or the price of the underlying security, the market (relative) value of the $\delta$-strangle in comparison to its BSM (relative) price. In fact, the explicit and simple expression for this measure and bound allows us to also study in detail the strangle's exit strategy and the corresponding optimal choice for a value of delta.
\end{abstract}

Keywords: call-put parity, option pricing, the Black-Scholes-Merton model, European options

\section{Introduction}

Options, as asset's price derivatives, are the primary tools available to the market participants for hedging their portfolio from directional risk and/or volatility risk. The so-called option's delta, which typically is denoted as $\delta$ or $\Delta$, measures the 'sensitivity' of the option's price to changes in the price of the underlying security, is the primary parameter one considers when using an option to mitigate directional risk. The option's delta is seen as the hedging ratio and is often also used (near expiration) by market participants as a surrogate to the probability that the option will expire in the money. With standard option pricing model of Black \& Scholes (1973) and Merton (1973), (abbreviated here as the BSM model, see below), these probabilities are readily available for direct calculations under the governing log-normality assumption of the asset's returns. Roughly speaking, a trader that sells (or buys) a put option at a strike located one standard deviation below the current asset's price, ends up with a 16-delta put contract option (i.e. with $\delta=-0.16)$. We denote the corresponding strike for this 16 -delta put contract option as $k_{0.16}^{-}$. Similarly, a trader that sells (or buys) a call option at a strike located one standard deviation above the current asset's price, ends up with a 16-delta call option (i.e. $\delta=0.16$ ). We denote the corresponding strike for this 16 -delta call contract as $k_{0.16}^{+}$. Thus, the corresponding strangle, which is obtained by selling a (negative) 16-delta put option and a (positive) 16-delta call option, is a delta-neutral strategy that is associated, very roughly, with a 0.68 probability for the asset's price to remain between the two strikes, $k_{0.16}^{-}$and $k_{0.16}^{+}$by expiration, all as resulting from the governing normal distribution assumption. We refer to such a strangle as a 16-delta Symmetric Strangle, only to indicate the common (absolute) delta value ( $\delta=0.16$ ) of its put and call components.

In a similar fashion we use the term a $\delta$-Symmetric Strangle to indicate the strangle obtained, for some fixed $\delta \in(0,0.5)$, from buying (or selling) a $\delta$-units put and a $\delta$-units call option contracts at the corresponding strikes $k_{\delta}^{-}$and $k_{\delta}^{+}$, respectively. Such a strangle would be a delta-neutral strategy offering zero directional risk but potentially useful for mitigating volatility risk ${ }^{1}$. We further denote by $\Pi_{\delta}$ the price of (or the credit received from) such $\delta-$ Symmetric Strangle. In this paper, we study, for a given $\delta$, the value of this $\delta$-Symmetric Strangle relative to the

\footnotetext{
1 Sometimes such a strangle is referred to a zero-delta strangle to reflect the lack of any directional risk to it.
} 
width of the corresponding spread $\left(k_{\delta}^{+}-k_{\delta}^{-}\right)$, adjusted for its present value (PV). More precisely, for any a $\delta \in(0,0.5)$, we define the relative value of the corresponding $\delta$-Symmetric Strangle as

$$
R_{\delta}:=\frac{\Pi_{\delta}}{P V\left(k_{\delta}^{+}-k_{\delta}^{-}\right)} .
$$

In the main result of the paper, we show that under the standard BSM option pricing model, the strangle's relative value, $R_{\delta},(1)$, is independent of the price of the underlying security and is a function only of $\delta$ and the prevailing volatility used in the pricing model. In fact, as we will see in Theorem 1 below, for any given $\delta \in(0,0.5)$, we have $R_{\delta} \leq \Re_{\delta}$ where

$$
\mathfrak{R}_{\delta}=-\frac{\phi\left(z_{\delta}\right)}{z_{\delta}}-\delta_{-\frac{u^{2}}{2}}
$$

and where $\phi(\cdot)$ is the standard normal density $(p d f), \quad \phi(u)^{z_{\delta}}:=e^{-\frac{u^{2}}{2}} / \sqrt{2 \pi}$, and $z_{\delta} \equiv \Phi^{-1}(\delta)$, is usual $\delta^{\text {th }}$ percentile of the standard normal distribution, whose cumulative distribution function $(c d f)$ is $\Phi(z):=\int_{-\infty}^{z} \phi(u) d u$. We point out that since $\delta<0.5$, we have $z_{\delta}<0$ in expression (2) of $\Re_{\delta}$.

As an illustration, one quickly finds by utilizing (2) that the 16 -delta Symmetric Strangle has a relative value of $\mathfrak{R}_{0.16}=0.08467$ and that the 30 -delta Symmetric Strangle has a relative value of $\mathfrak{R}_{0.30}=0.36$. That is to say that under the standard BSM option pricing model, one would expect the price of the 30 -delta Symmetric Strangle to be at most $36 \%$ of the width of the spread between the corresponding strikes, irrespective of the security's price, or time to expiry, and irrespective of the prevailing volatility. More generally, it follows from Theorem 1, that for a any given $\delta \in(0,0.5)$, the corresponding $\delta$-strangle's price, $\Pi_{\delta}$, as calculated under the BSM pricing model, satisfies

$$
\Pi_{\delta} \leq \mathfrak{R}_{\delta} \times P V\left(k_{\delta}^{+}-k_{\delta}^{-}\right),
$$

irrespective of the security's price, or time to expiry, and irrespective of the prevailing volatility. We also illustrate how this measure $\mathfrak{R}_{\delta}$ in (2) may be used as a benchmark to assess the market pricing (or 'worthiness') of the $\delta$ symmetric strangle compared to its (relative) price, $R_{\delta}$, as suggested by standard BSM pricing model. The explicit expression of $\mathfrak{R}_{\delta}$ as is given in (2) allow us to also address, in the last section, the strangle's exit strategy and the corresponding optimal choice of $\delta$ for it. However, in the next section we first show how, for a fixed value of $\delta$, ths standard BSM option pricing model can be reparmetrized in terms of $\delta$ and a volatility parameter.

\section{Pricing the $L$-Unit Option Contract}

One of the most widely celebrated option pricing model for equities (and beyond) is that of Black and Scholes (1973). Their pricing model is derived under some simple assumptions concerning the distribution of the asset's returns, coupled with presumptive continuous hedging, zero dividend, risk-free interest rate, $r$, and no cost of carry or transactions fees. While the aptness of these assumptions has often been criticized (see for example, Yalincak (2012) or Bueno-Guerrero, (2019), it has remained as a leading option pricing model for the retail trading practitioner (e.g.: Sinclair (2010)). However, in its standard form, the BSM model evaluates, for a risky asset with a current market price $\mu$, the price of an European call option contract at a strike $k$ and $t$ days to expiration as:

$$
c_{\mu}(k)=\mu \times \Phi\left(d_{1}(k)\right)-k \cdot e^{-r t} \times \Phi\left(d_{2}(k)\right) .
$$

Here, using the standard notation,

$$
d_{1}(k):=\frac{\log \left(\frac{\mu}{k}\right)+\left(r+\frac{\sigma^{2}}{2}\right) t}{\sigma \sqrt{t}} \quad \text { and } \quad d_{2}(k):=d_{1}(k)-\sigma \sqrt{t},
$$

where $\sigma$ denotes the standard deviation of the daily asset's returns, and $\Phi(\cdot)$ is the standard normal cdf defined above. The model for the corresponding price of a put option contract, $p_{\mu}(k)$, may be obtain from expression (3) of $c_{\mu}(k)$, by exploiting the so-called put-call parity which is expressed by the equation

$$
\mu-c_{\mu}(k)=k \cdot e^{-r t}-p_{\mu}(k)
$$

see for example Jiang (2005, Theorem 2.3) or Peskir and Shiryaev (2002) for additional details. This parity implies that the price of the corresponding put option contract is,

$$
p_{\mu}(k)=k \cdot e^{-r t} \times \Phi\left(-d_{2}(k)\right)-\mu \times\left[1-\Phi\left(d_{1}(k)\right)\right] .
$$

There is substantial body of literature dealing with the BSM option pricing model in (3)-(6), its refinements, its extensions and the so-called, its implied 'Greeks' (i.e. the various partial derivatives of different orders, representing the model's "sensitivities" to changes in its parameters). The interested reader is referred to standard textbooks such as Wilmott, Howison, Dewynne (1995), Hull (2005), Jiang (2005) or Iacus (2011).

As we already mentioned in the Introduction, we focus our attention here on the option's delta, which we denote by $\Delta$ as a function with a corresponding value of $\delta \in(0,1)$. More specifically, while suppressing (for sake of simplicity 
for now) from the notation $r, t$ and $\sigma^{2}$, we define for the call and the put contracts options their respective $\Delta$ functions as, $\Delta_{c}(k):=\partial c_{\mu}(k) / \partial \mu$ and $\Delta_{p}(k):=\partial p_{\mu}(k) / \partial \mu$. It follows immediately from the put-call parity equation in (5) that $\Delta_{p}(k)=-\left(1-\Delta_{c}(k)\right)$. It is well known (see for Example, Jiang (2005)) that for the BSM pricing model in (3), $\Delta_{c}(k)=\Phi\left(d_{1}(k)\right)$, where $d_{1}(k)$ is given in (4), and hence $\Delta_{p}(k)=-\left(1-\Phi\left(d_{1}(k)\right) \equiv-\Phi\left(-d_{1}(k)\right)\right.$.

For its supreme importance to portfolio hedging, the investor/trader often needs to buy (or sell) an option at a strike, $k$, which is associated with a specified and desired value $\delta$ of the option's $\Delta$. For any given $\delta \in(0,1)$, we let $k_{\delta}^{+}$ denote the (unique) solution of the equation $\Delta_{c}\left(k_{\delta}^{+}\right)=\delta$, or equivalently the solution of

$$
\Phi\left(d_{1}\left(k_{\delta}^{+}\right)\right)=\delta \text {. }
$$

Accordingly, it follows immediately that $k_{\delta}^{+}$satisfies the equation

$$
d_{1}\left(k_{\delta}^{+}\right)=\Phi^{-1}(\delta) \equiv z_{\delta},
$$

and hence, by utilizing (4) in (8) leads to the solution as

$$
k_{\delta}^{+}=\mu \cdot e^{-z} \delta^{v+v^{2} / 2+r t},
$$

where we have substituted $v \equiv \sigma \sqrt{t}$ throughout. It should be clear from (9) that if $\delta<0.5$, one has $z_{\delta}<0$ and therefore $k_{\delta}^{+}>\mu$, so that the corresponding call option is said to be 'out of the money' (OTM). Also, note that it follows from (4) and (8) that $d_{2}\left(k_{\delta}^{+}\right)=d_{1}\left(k_{\delta}^{+}\right)-\sigma \sqrt{t} \equiv z_{\delta}-v$, so that

$$
\Phi\left(d_{2}\left(k_{\delta}^{+}\right)\right)=\Phi\left(z_{\delta}-v\right)
$$

in (3). Indeed, with the re-parameterization by $(\delta, v)$ (with $v \equiv \sigma \sqrt{t}$ ), of the BSM option pricing model in (3), we may re-express, upon using the matching expressions (7)-(10) in equation (3), the current price of a $\delta$-unit call option in a much simpler form as

$$
\begin{aligned}
c_{\mu}(\delta, v) \equiv c_{\mu}\left(k_{\delta}^{+}\right)= & \mu \times \delta-k_{\delta}^{+} \cdot e^{-r t} \times \Phi\left(z_{\delta}-v\right) \\
& =\mu \times\left[\delta-e^{-z} \delta^{v+v^{2} / 2} \times \Phi\left(z_{\delta}-v\right)\right],
\end{aligned}
$$

for any $\delta \in(0,1)$ and with $v>0$.

Remark 1: Note in passing that in practice, the option's $\delta$ is often used as a crude approximation to the probability the option will end in the money, $\operatorname{Pr}(I T M)$, which by (10), (11) is equal to $\Phi\left(d_{2}\left(k_{\delta}^{+}\right)\right) \equiv \Phi\left(z_{\delta}-v\right)$. However, since $v \equiv \sigma \sqrt{t}>0$, it immediately follows that $\left.\left.\Phi\left(z_{\delta}-v\right)\right) \leq \Phi\left(z_{\delta}\right)\right) \equiv \delta$. Hence, for any $\delta \in(0,1)$ and $v>0, \operatorname{Pr}(I T M) \leq \delta$ and only near expiration, as $\lim _{t \rightarrow 0} \operatorname{Pr}(I T M)=\delta$, it holds.

Similarly to (11), we calculate the current price of the $\delta$-unit put contract option by using the put-call parity equation in (5), and by noting that by (7) the corresponding $k_{\delta}^{-}$strike for the put contract is the same as the strike $k_{1-\delta}^{+}$of the $(1-\delta)$ - unit call option contract, so that $k_{\delta}^{-} \equiv k_{1-\delta}^{+}$. Accordingly, since $z_{\delta} \equiv-z_{1-\delta}$, we obtain from (9) that

$$
k_{\delta}^{-}=\mu \cdot e^{z} \delta^{v+v^{2} / 2+r t} .
$$

Hence, it follows immediately from (5) and (12), that under the $(\delta, v)$ re-parameterization, the expression for the current price of the $\delta$-unit put option is,

$$
\begin{aligned}
p_{\mu}(\delta, v) \equiv p_{\mu}\left(k_{\delta}^{-}\right) & =-\delta \mu+k_{\delta}^{-} \cdot e^{-r t} \times\left(1-\Phi\left(z_{1-\delta}-v\right)\right) \\
& =-\mu \times\left[\delta-e^{z} \delta^{v+v^{2} / 2} \times \Phi\left(z_{\delta}+v\right)\right] .
\end{aligned}
$$

It should be clear from (12) that if $\delta<0.5$ and $r=0$, one has $z_{\delta}<0$ and therefore $k_{\delta}^{-}<\mu$ only if $v<-2 \cdot z_{\delta}$, in which case, the corresponding put option is said to be 'out of the money' (OTM). Hence, we will restrict our attention to the practical case of the above parametrization in which $(\delta, v)$ are such that $k_{\delta}^{-}<\mu<k_{\delta}^{+}$, or alternatively, $(\delta, v) \in B$, where

$$
\mathrm{B}=\{(\delta, v) ; \delta>0, \& v>0 \text {, s.t. } \delta<\Phi(-v / 2)\} .
$$

We further point out that the two strikes, $k_{\delta}^{+}$and $k_{\delta}^{-},\left(\equiv k_{1-\delta}^{+}\right)$, need not be symmetrical with respect of the current price $\mu$ of the underlying security (i.e.: $\mu-k_{\delta}^{-} \neq k_{\delta}^{+}-\mu$ ). It is well-known that the occasional observed asymmetry of these equal $\delta$ - units strikes is a fixture of the skew in the volatility surface that is affecting the option pricing model (see for example Gatheral (2006), or Doran \& Krieger (2010)).

\section{The Relative Value of the $\mid-$-Symmetric Strangle}

Consider now a trader that desires to simultaneously sells (say), at some given level of $\delta<0.5$, the $\delta$-unit put and 
the $\delta$-unit call contracts so as to form the 'OTM' $\delta$-Symmetric Strangle strategy. The total selling price of this strangle as calculated under the BSM pricing model, is therefore $\Pi_{\delta}=c_{\mu}\left(k_{\delta}^{+}\right)+p_{\mu}\left(k_{\delta}^{-}\right)$. As a measure for assessing the 'worthiness' of this strangle, we consider the 'value' of the selling price, $\Pi_{\delta}$, relative to the present value of the spread between the strikes, namely, $P V\left(k_{\delta}^{+}-k_{\delta}^{-}\right)=\left(k_{\delta}^{+}-k_{\delta}^{-}\right) \times e^{-r t}$. We express this relative value measure in (1) as

$$
R(\delta, v):=\frac{\Pi_{\delta}}{P V\left(k_{\delta}^{+}-k_{\delta}^{-}\right)}=\frac{c_{\mu}(\delta, v)+p_{\mu}(\delta, v)}{\left(k_{\delta}^{+}-k_{\delta}^{-}\right) \times e^{-r t}} .
$$

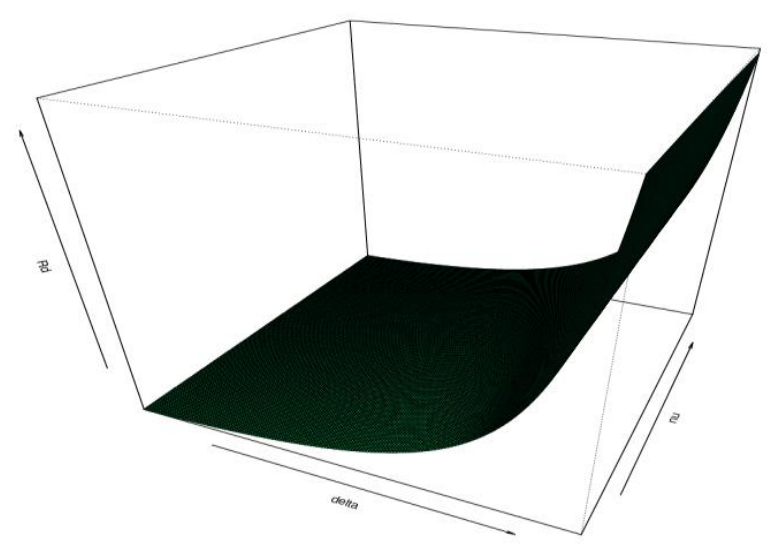

Figure 1. The Relative Value function $R(\delta, v)$ of the $\delta$-Symmetric Strangle for $\delta<0.5$ and $v \in(0,1)$.

Note that by its definition, $R(\delta, v) \geq 0$ for all $\delta \in(0,0.5)$ and $v>0$, in particular over the set B. Further, since an European option price is (linearly) homogeneous in $\mu$, and in the strike, $k$, (see Theorem 6 of Merton (1973)), the ratio $R(\delta, v)$ in (14), is independent of the current price of the underlying security, $\mu$. Also note that since we account in (14) for the present value of the spread between the strikes, this quotient is, by construction, also independent of the risk-free interest rate, $r$. This can fully realized by substituting expressions (9), (11), (12) and (13) in $R(\delta, v)$ and simplifying the resulting terms, to obtain, for each $\delta<0.5$ and $v \equiv \sigma \sqrt{t}>0$, the expression,

$$
R(\delta, v)=\frac{e^{z} \delta^{v} \cdot \Phi\left(z_{\delta}+v\right)-e^{-z} \delta^{v} \cdot \Phi\left(z_{\delta}-v\right)}{e^{-z} \delta^{v}-e^{z} \delta^{v}},
$$

for the relative value of the $\delta$-Symmetric Strangle under the BSM option pricing model. We point that the values of $R(\delta, v)$ in (15) are straightforward to calculate for any $(\delta, v)$.

Figure 1 above provides the graph of $R(\delta, v)$ for various values of $(\delta, v)$, with $0<\delta<0.5$ and $0<v<1$, where $v=\sigma \sqrt{t}$ representing realistic values for $t$ (the time in days to expiry) and the model's daily (implied or historical) volatility, $\sigma$. In any case, the properties of $R(\delta, v)$, as a function of $\delta$ and $v$ (in $B$ ) are of interest. In the Appendix below we show that for a fixed $\delta<0.5, R(\delta, v)$ is monotonically non-increasing function of $v$ (with $\partial R / \partial v \leq 0)$ and that for a fixed $v>0, R(\delta, v)$ is monotonically increasing function of $\delta$ (with $\partial R / \partial \delta>0$ ). In Theorem 1 below we present the main results of the paper, establishing that $R(\delta, v) \leq \mathfrak{R}_{\delta}$, and claculating expicitly the value of the bound $\mathfrak{R}_{\delta}$. The proof of the Theorem is provided in the Appendix.

Theorem 1 Under the BSM model and irrespective of the current price, $\mu$, of the underlying security, the current risk-free interest rate, $r$, and irrespective of the time to expiry, $t$, and the presumed volatility (either implied or historical), the upper bound to the relative value $R(\delta, v)$, of the OTM $\delta$-Symmetric Strangle with $\delta \in(0,0.5)$, depends only on $\delta$ and is given by, $0<R(\delta, v) \leq \mathfrak{R}_{\delta}$, where

$$
\mathfrak{R}_{\delta}:=\lim _{v \rightarrow 0} R(\delta, v)=-\frac{\phi\left(z_{\delta}\right)}{z_{\delta}}-\delta .
$$

Moreover, $\lim _{\delta \rightarrow 0} \Re_{\delta}=0$, and for all $\delta \in(0,0.5)$,

$$
\mathfrak{R}_{\delta}^{\prime}:=\frac{d}{d \delta} \Re_{\delta}=\frac{1}{z_{\delta}^{2}}>0 .
$$

The results of Theorem 1 and the bound $\mathfrak{R}_{\delta}$ in (16) provide a benchmark for assessing the value, in relative terms, of a 
$\delta$-Symmetric Strangle under the BSM option pricing model in (3)-(6), as applicable to any security (i.e., independent of the current underlying security price $\mu$ ), to any expiry (i.e., independent of $t$ ), and under any presumed volatility (i.e., independent of $\sigma$ ). In fact, if $\hat{R}_{\delta}$ denotes the market (relative) value of a $\delta$-Symmetric Strangle (that is, the market version of (1)), then, this strangle would be deemed 'well-priced' compared to its (relative) price under the BSM option pricing model, as long as $\hat{R}_{\delta} \geq \mathfrak{R}_{\delta}$. In Figure 2 below, we graph the values of this function, $\mathfrak{R}_{\delta}$ (in (16) or (2)) for all $0<\delta<0.5$.

Remark 2: The results stated in Theorem 1 and their derivations are valid in the BSM 'world', in which the distribution of the asset's returns assumed to have a constant variability throughout and do not take into account the volatility 'skew' or 'smile' that is often being observed by the traders across the discretized options' grid equipped with bid-ask price spreads. It surely implies that the BSM pricing model (with all it inputs) undervalues the $\delta$-Symmetric Strangle, whenever $\mathfrak{R}_{\delta}<\hat{R}_{\delta}$, where $\hat{R}_{\delta}$ is its market (relative) value (i.e. the market version of $(1)$ ).

Illustration: As an illustration of it's usage, consider the market EOD (end of day) market pricing of IBM (International Business Machine Corp.) as of May 13th, 2020. We find that the 34-delta symmetric strangle for the June 5th, 2020 expiration with the strikes of $k_{1}=\$ 112$ and $k_{2}=\$ 120$ for the sold put and call, respectively, has a market mid-price of $\hat{\Pi}_{0.34}=\$ 4.60$ (along with current ticker price of $\mu=\$ 115.73$, with $t=23$ days to expiration, and $I V=38.32 \%$ (average) implied volatility, so that $\sigma=I V / \sqrt{365}=0.0200576$ ). This results with a market relative value of

$$
\hat{R}_{0.34}:=\frac{\hat{\Pi}_{0.34}}{\left(k_{2}-k_{1}\right)}=\frac{4.60}{(120-112)}=0.575,
$$

for this 34-delta Strangle in IBM, whereas, by using (2), we calculate under the BSM pricing model a relative value of $\mathfrak{R}_{0.34}=0.548$ for this 34-delta strangle. Thus, the BSM pricing model (with its constant variance assumption, etc.) under-values this strangle (in relative terms) as compared to its actual market value. Similar result is obtained with the relative value of a 21-delta strangle with 100 days to expiration in BA (Boeing Co.), which yields $\hat{R}_{0.21}=0.156$ as compared to $\Re_{0.21}=0.147$, see Table 1 below.

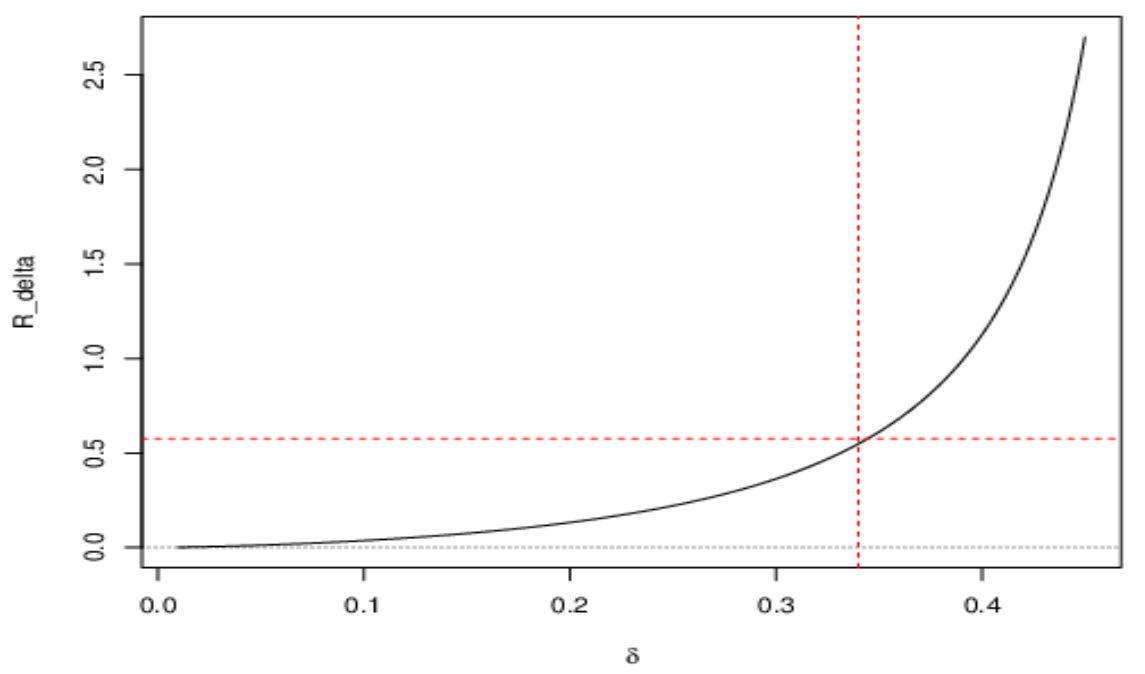

Figure 2. The relative value $\mathfrak{R}_{\delta}$ as a function of $\delta$. Marked in red is the market relative value (current, as of EOD, May, 13th, 2020), $\hat{R}_{0.34}=0.575$, of a 34-delta symmetric strangle with strikes $\$ 112$ and $\$ 120$ in IBM (see Example 1 and Table 1 for more details.)

Also included in the table the market pricing of $\delta$ - symmetric strangles for additional securities, with different $\delta$, underlying prices, $I V$ and days to expiration. In all cases listed in the Table, the market (relative) value $\hat{R}_{\delta}$ exceeded that of the corresponding BSM (relative) value $\mathfrak{R}_{\delta}$. Thus, in these noted cases, the BSM pricing model (with all its inputs) appears to undervalue the strangles (in relative terms) as compared to their market (relative) value. The reader is 
invited to check the validity of Theorem 1 results and the applicability of the bound $\Re_{\delta}$ in (16) as a benchmark for the market pricing (in relative value) of a $\delta$-Symmetric Strangle with any other traded security options at any expiration.

Table 1. Oserved market relative values $\hat{R}_{\delta}$ of the $\delta$-Symmetric Strangle

\begin{tabular}{cccccccccc}
\hline Ticker & $\mu$ & \multicolumn{1}{c}{$\boldsymbol{I V}$} & Days & $\delta$ & $k_{1}$ & $k_{2}$ & $\hat{\Pi}_{\delta}$-Price & $\hat{R}_{\delta}$ & $\mathfrak{R}_{\delta}$ \\
\hline SPY & 281.60 & 0.3529 & 37 & 0.170 & 250 & 302 & 5.19 & 0.107 & 0.095 \\
LLY & 157.93 & 0.3597 & 156 & 0.200 & 130 & 185 & 8.22 & 0.150 & 0.133 \\
BA & 121.50 & 0.7685 & 100 & 0.210 & 95 & 175 & 12.45 & 0.156 & 0.147 \\
TLT & 168.50 & 0.2029 & 16 & 0.255 & 162 & 170 & 1.97 & 0.246 & 0.232 \\
C & 40.60 & 0.6851 & 219 & 0.295 & 32 & 52.5 & 7.05 & 0.362 & 0.345 \\
IBM & 115.73 & 0.3832 & 23 & 0.340 & 112 & 120 & 4.60 & 0.575 & 0.548 \\
GOOG & 1349.33 & 0.3356 & 65 & 0.405 & 1320 & 1400 & 102.65 & 1.283 & 1.207 \\
\hline
\end{tabular}

Description: Values of $\hat{R}_{\delta}$ are provided for various tickers and durations as were priced on EOD "2, May 13, 2020, and are compared to the bound $\mathfrak{R}_{\delta}$ (16) calculated under the BSM option pricing model.

The empirical (market) results displayed in Table 1, illustrate the tremendous practical and strategy implications the results of Theorem 1 have. With such theoretical results at hand, retail traders and and market participants are now able to quickly assess whether or not the strangle they buy (or sell) is overpriced or under-priced in the market as compared to its Black-Scholes price, regardless the market volatility, the duration of the contract or the underlying assest's price . It provides for a common benchmark for assessing and comparing the market (relative) pricing of a major trading strategy (namely the $\delta$-Symmetric Strangle) across various securities and assets, across various durations and irrespective of the underlying security-specific volatility (implied or historical).

\section{Strategizing}

One of the appealing aspects of a $\delta$-Symmetric Strangle is that from the outset, it is a delta-neutral strategy with zero directional risk, initially. Moreover a trader that sells such a strangle, for some fixed $\delta<0.5$, at the corresponding two strikes $k_{\delta}^{-}$and $k_{\delta}^{+}$, benefits from a well defined probability of success, that may be calculated under the current distribution of the asset's returns implied by BSM option pricing model in (3) and (6). Specifically, for a given value $\delta<0.5$ and $v>0$, the initial probability that the underlying security price would remain, at expiration, between $k_{\delta}^{-},\left(\equiv k_{1-\delta}^{+}\right)$and $k_{\delta}^{+}$is simply (see Remark 1$)$,

$$
\alpha \equiv \Phi\left(-z_{\delta}-v\right)-\Phi\left(z_{\delta}-v\right) .
$$

Hence, the expected reward for a trader that sells the strangle for $\Pi_{\delta}=c_{\mu}\left(k_{\delta}^{+}\right)+p_{\mu}\left(k_{\delta}^{-}\right)$(as credit) and plans to exit and buy it back for a fraction $\lambda \in(0,1]$ of the credit received is

$$
E_{\lambda}(\delta):=\alpha \Pi_{\delta}-(1-\alpha) \lambda \Pi_{\delta} .
$$

In relative terms, this expected reward, relative to the present value of the spread between the strikes, becomes

$$
\bar{E}_{\lambda}(\delta):=\frac{E_{\lambda}(\delta)}{P V\left(k_{\delta}^{+}-k_{\delta}^{-}\right)} \leq \alpha \cdot \Re_{\delta}-(1-\alpha) \lambda \cdot \Re_{\delta},
$$

where $\mathfrak{R}_{\delta}$ is given in (2). As was mentioned in the introduction and pointed out in Remark 1, for small values of $v$ (i.e. near expiration) we may approximate the 'success' probability in (18) as $\alpha \approx(1-2 \delta)$.

\footnotetext{
${ }^{2}$ Source: EOD market pricing were obtained using the TOS platform of TDAmeritrade
} 


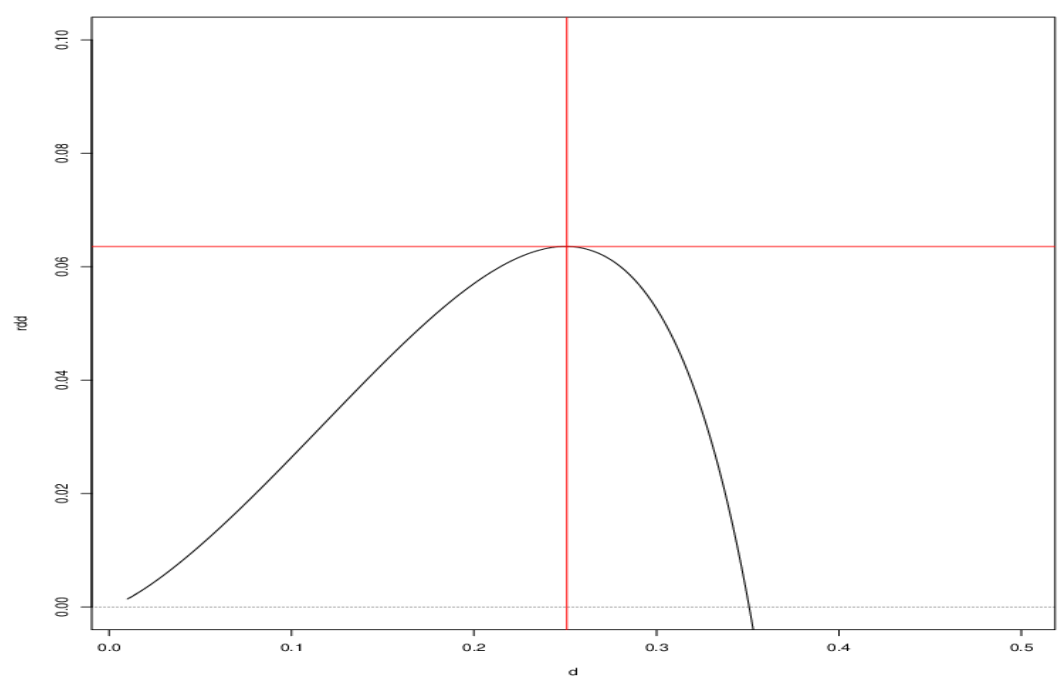

Figure 3. The expected relative reward function, $E_{\lambda}(\delta)$ as a function of $\delta$ with $\lambda=0.5$. The maximal value is achieved at $\delta^{*}=0.2336$ at which point, $E_{\lambda}\left(\delta^{*}\right)=0.05615$.

Accordingly, for any given fraction $\lambda \in(0,1]$ the expected relative reward in (19), under this approximation would be,

$$
E_{\lambda}(\delta)=(1-2 \delta(1+\lambda)) \times \Re_{\delta} .
$$

Observe that $E_{\lambda}(\delta) \geq 0$ as long as $\delta \leq 1 / 2(1+\lambda)$ and that, upon using (16) and (17), the equation

$$
E_{\lambda}^{\prime}(\delta) \equiv-2(1+\lambda) \Re_{\delta}+(1-2 \delta(1+\lambda)) \Re_{\delta}^{\prime}=0,
$$

is seen to have a unique root, $\delta^{*}$, at which point $E_{\lambda}(\delta)$ attains its maximal value. That is, for a given fraction $\lambda \in(0,1]$, this root $\delta^{*}=h(\lambda)$, must solves the equation

at which point $E_{\lambda}^{*}:=E_{\lambda}\left(\delta^{*}\right) \geq E_{\lambda}(\delta)$.

$$
\delta\left(1-z_{\delta}^{2}\right)-z_{\delta} \phi\left(z_{\delta}\right)=\frac{1}{2(1+\lambda)},
$$

In Figure 3 we present, as an illustration, the graph of the relative reward function, $E_{\lambda}(\delta)$, for a trader who sells a $\delta-$ Symmetric Strangle, and wishes, as a matter of strategy, to exit it upon a loss of $50 \%$ of the credit received. This case corresponds to $\lambda=0.5$ and results with an optimal choice for $\delta$ of $\delta^{*}=0.2336$ for this strategy to yield a maximal expected relative reward of $E_{0.5}^{*}=0.05615$.

In Table 2, we provide the 'optimal' values for $\delta$ as were calculated (as a numerical solution of (21)) for various choices of $\lambda$, along with the corresponding values of the maximal expected reward $E_{\lambda}^{*}$, and the matching initial probability of "success" of this $\delta^{*}-$ Symmetric Strangle strategy. As can be seen from Table 2, the selling of a 'standard' 16-delta symmetric strangle with its $\alpha\left(\delta^{*}\right)=0.67$ 'success' probability should be coupled with an exit strategy that limits losses at $100 \%$ of the credit received may yield a maximal expected relative reward of $E_{1}^{*}=0.031$.

In contrast, the selling of a 30-delta symmetric strangle of the lesser 'success' probability (of $\alpha\left(\delta^{*}\right)=0.4$ ) should be coupled with an exit strategy that limits losses at $25 \%$ of the credit received, but may triple the maximal expected reward to $E_{0.25}^{*}=0.091$. 
Table 2. Optimal choice of $\delta$

\begin{tabular}{cccc}
\hline$\lambda$ & $\delta^{*}$ & $E^{*}$ & $\alpha\left(\delta^{*}\right)$ \\
\hline 0.25 & 0.300 & 0.091 & 0.400 \\
0.40 & 0.256 & 0.067 & 0.489 \\
0.50 & 0.234 & 0.056 & 0.533 \\
0.60 & 0.216 & 0.048 & 0.567 \\
0.75 & 0.194 & 0.040 & 0.611 \\
1.00 & 0.164 & 0.031 & 0.670 \\
\hline
\end{tabular}

Description: The optimal choice for $\delta$ for the $\delta$ - Symmetric Strangle strategy, calculated for 'exits' with the various fractional loss $\lambda$.

\section{Conclusion}

We presented new measure of the relative value of a $\delta$ - Symmetric Strangle and provided for its explicit expression as calculated under the standard BSM option pricing model. This measure accounts for the price of the strangle, relative to the Present Value of the spread between the two strikes, all expressed, after a natural re-parameterization, in terms of delta and a volatility parameter. It was shown that under the standard BSM model, this measure of relative value is bounded by a simple function of $\delta$ only and is independent of the time to expiry, the price of the underlying security or the prevailing volatility used (either implied or historical) in the pricing model. As was stated in Remark 2, this result is only valid in the standard BSM 'world' in which the distribution of the asset's returns assumed to have a constant variability throughout. However, we demonstrated how this bound can be used as a useful benchmark to assess, regardless the market volatility, the duration of the contract or the price of the underlying security, the market (relative) value of the $\delta$-strangle in comparison to its BSM (relative) price. Moreover, the explicit and simple expression for this measure and bound allows us to also provide in detail an exit strategy and the corresponding optimal choice for a value of delta.

\section{References}

Black F., \& Scholes M. (1973). The pricing of options and corporate liabilities. The Journal of Political Economy, 637-654. https://doi.org/10.1086/260062

Bueno-Guerrero, A. (2019). Black-Scholes and Heston Models with Stochastic Interest Rates and Term Structure of Volatilities, The Journal of Derivatives, Fall 2019, 27(1), 32-48. https://doi.org/10.3905/jod.2019.1.078

Doran, J., \& Krieger, K. (2010). Implications for Asset Returns in the Implied Volatility Skew. Financial Analysts Journal, 66(1), 65-76. https://doi.org/10.2469/faj.v66.n1.9

Gatheral, J. (2006). The Volatility Surface, John Wiley and Sons, NJ.

Hull, C. J. (2017). Options, Futures and Other Derivatives, 10th Ed, Pearson, NY.

Iacus, M. S. (2011). Option Pricing and Estimation of Financial Models with R, John Wiley and Sons, NY. https://doi.org/10.1002/9781119990079

Jiang, L. (2005). Mathematical Modeling and Methods of Option Pricing, Translated from Chinese by Li. C, World Scientific, Singapore. https://doi.org/10.1142/5855

Merton, R. (1973). Theory of rational option pricing. The Bell Journal of Economics and Management Science, 141-183.

Peskir, G., \& Shiryaev, A. (2002). A Note on the Call-Put Parity and a Call-Put Duality. Theory of Probability and Its Applications , 46(1), 167-170. https://doi.org/10.1137/S0040585X97978841

Sinclair, E. (2010). Option Trading; Pricing and Volatility Strategies and Techniques, John Wiley and Sons, NJ

Wilmott, P., Howison, S., \& Dewynne, J. (1995). The Mathematics of Financial Derivatives: A Student Introduction, 1st Ed, Cambridge University Press. https://doi.org/10.1017/CBO9780511812545

Yalincak, H. O. (2012). Criticism of the Black-Scholes Model: But Why is It Still Used?: (The Answer is Simpler than the Formula). https://doi.org/10.2139/ssrn.2115141 


\section{Appendix}

In this appendix we provide the proof of Theorem 1 along with an auxiliary result regarding the coordinate-wise behavior of $R(\delta, v)$ as given in (15) over the practical domain B. To begin with, note first that since

$$
e^{z \delta^{v+v^{2} / 2}} \equiv \frac{\phi\left(z_{\delta}\right)}{\phi\left(z_{\delta}+v\right)} \quad \text { and } \quad e^{-z \delta^{v+v^{2} / 2}} \equiv \frac{\phi\left(z_{\delta}\right)}{\phi\left(z_{\delta}-v\right)},
$$

we may express $R(\delta, v)$ in (15), entirely in terms of the standard normal $p d f$ and $c d f$, as

$$
R(\delta, v)=\frac{\phi\left(z_{\delta}-v\right) \cdot \Phi\left(z_{\delta}+v\right)-\phi\left(z_{\delta}+v\right) \cdot \Phi\left(z_{\delta}-v\right)}{\phi\left(z_{\delta}+v\right)-\phi\left(z_{\delta}-v\right)} .
$$

Upon differentiating expression (22) of $R(\delta, v)$, with respect to $\delta$ and with respect to $v$ along with the fact that $\phi^{\prime}(u):=\frac{d}{d u} \phi(u)=-u \phi(u)$ we obtain the following results.

Lemma 1 With $R(\delta, v)$ as defined in (15) above we have,

- For each fixed $\delta<0.5$ (so that $\left.z_{\delta}<0\right), \frac{\partial R}{\partial v}=\frac{2 a}{b^{2}}\left(b+z_{\delta} \cdot d\right) \leq 0$,

- For each fixed $v>0, \frac{\partial R}{\partial \delta}=-\left(z_{\delta}-v\right) \cdot a \cdot d>0$,

where, $d:=\left(\Phi\left(z_{\delta}+v\right)-\Phi\left(z_{\delta}-v\right)\right)>0, b:=\left(\phi\left(z_{\delta}+v\right)-\phi\left(z_{\delta}-v\right)\right)>0, \quad$ and $a:=\phi\left(z_{\delta}+v\right) \cdot \phi\left(z_{\delta}-v\right)>0$.

Proof of Lemma 1: The proofs of these results, though tedious, are straightforward to establish noting that $\left(z_{\delta}-v\right)<0$ and $\left(b+z_{\delta} \cdot d\right) \leq 0$ over $\mathrm{B}$; the details are omitted.

We conclude with a proof of our main results.

Proof of Theorem 1: That $R(\delta, v)$ is a monotonically decreasing function of $v$ for each fixed $\delta \in(0,0.5)$ is seen by direct calculation, $\partial R(\delta, v) / \partial v \leq 0$ (by Lemma 1). The results stated in (16) follow immediately by a straightforward application of L'Hopital's rule to the numerator and denominator that comprise expression (15) of $R(\delta, v)$ and noting that it trivially also independent of $\mu$ and $r$ by construction. By another direct application of L'Hopital's rule to the quotient $\phi\left(z_{\delta}\right) / z_{\delta}$ along with the facts that $\frac{d}{d \delta} \phi\left(z_{\delta}\right)=-z_{\delta} \phi\left(z_{\delta}\right) z_{\delta}^{\prime}$ and $z_{\delta}^{\prime}=1 / \phi\left(z_{\delta}\right)$ leads to the second assertion as well as to the result stated in (17).

\section{Copyrights}

Copyright for this article is retained by the author(s), with first publication rights granted to the journal.

This is an open-access article distributed under the terms and conditions of the Creative Commons Attribution license which permits unrestricted use, distribution, and reproduction in any medium, provided the original work is properly cited. 